\title{
Characterisation of crack development in bimrocks using digital image correlation
}

\author{
Mansour Sharafisafa ${ }^{1}$, Zeinab Aliabadian ${ }^{2}$, and Luming Shen ${ }^{1}$ \\ ${ }^{1}$ The University of Sydney \\ ${ }^{2} \mathrm{UNSW}$
}

June 8, 2020

\begin{abstract}
Bimrocks feature stiff blocks randomly distributed in a soft matrix. Despite their wide distribution in nature, their significant effects on the failure of rock structures such as slopes, mines and tunnels have not been fully understood. Bimrocks behave differently as compared with other rock types since they contain mix of blocks of different strength, size and volume distribution and a matrix of different physical and mechanical characteristics. The purpose of this study is to investigate deformation behaviour and crack development trends of bimrocks containing blocks of different sizes (small $<1 \mathrm{~mm}$, medium $2-4 \mathrm{~mm}$, and large $5-8 \mathrm{~mm}$ ). The Brazilian disc test is performed with the digital image correlation (DIC) technique being employed for deformation analysis. It is found that the size of blocks significantly influences the deformation and failure behaviour of a bimrock. Bimrocks with large blocks exhibit both tensile cracks parallel to the loading direction and shear cracks inclined towards the loading direction. Moreover, the development of fracture process zone (FPZ) is highly dependent upon block size. In particular, bimrocks with large blocks exhibit obvious development of FPZ, while no FPZ is observed for those with small blocks. In fact, the specimens with small blocks cannot be called a bimrock since they behave similar to a homogenous intact disc specimen in which a crack splits the disc into two halves in a typical Brazilian test.
\end{abstract}

\section{Hosted file}

Manuscript-FFEMS.docx available at https://authorea.com/users/330648/articles/457949characterisation-of-crack-development-in-bimrocks-using-digital-image-correlation 